\section{Discrete charges in quantum mechanics}

Gravitation, Electromagnetism and Quantized Charge: The Einstein Insight. By Daniel M. Pisello. Pp. 94. (Ann Arbor Science: Ann Arbor, Michigan, 1979.) Distributed in UK, Africa and Europe by Wiley: Chichester, UK. £7.55.

BY means of dogmatic pronouncements on the limitations of human imagination and language, the quantum mechanical philosophy effectively takes away from the younger physicists their right to question and create. If one finds it necessary to question or theorize beyond the restraints so successfully built into the quantum mechanical rationale, one is then, by fiat, pejoratively considered a revolutionary". Thus ends Mr Pisello's thin treatise, half philosphical, half mathematical, and always bitter. This work will not revolutionise physics, "pejoratively" or otherwise. The book's interest, however, lies in the way its author attempts to link his philosophical and political attitudes with a mathematical search for a purely field theoretical approach to the existence of discrete charges in quantum mechanics. The result is an exercise in topology elevated to the rank of "unified theory" through a casual parallel with Einstein's own epistemological objections to the theory of quantum mechanics.

The first three chapters present the conceptual difficulties arising from scientific breakthroughs like the theories of special relativity, general relativity and quantum mechanics. Old and new concepts which apparently contradict each other are reviewed, the limitations inherent in the use of a language created within an earlier framework are discussed, and the need for a "principle of complementarity" is challenged on philosophical grounds. A rapid analysis of the double slit experiment for which "neither linear wave nor discrete particle interpretation provides an adequate representation of the transfer of energy from source to detector' 'is used to justify searching for the "basic nature of the field variable". The remainder of the book is devoted to this search in the form of mathematical exercises in homotopy theory and general relativity. It ends with a succinct theory of charge as a counting procedure for "an identifiable number of separate left- and right-hand doughnuts"'.

Noticing that physical field theories deal only with continuous entities while the physical world displays basic discontinuities (the elementary electric charge of the electron being the one specific case considered here) is not new, but the whole preoccupation with continuity, infinities and singularities is indeed continuing. In this sense Mr Pisello's book is part of the contemporary mood. To try to save continuity by hiding the "inside" of particles into topology has been done with mastery by Professor John Wheeler creating "wormholes" for the delight of many students of general relativity. $\mathrm{Mr}$ Pisello attempts something similar but each piece of his puzzle unfortunately remains physically and also mathematically quite disconnected. At the Einstein centennial celebration at UNESCO in Paris a few weeks ago, Professor Dirac stressed his own dis-satisfaction with quantum mechanical calculations for which we "learned to turn a disinterested eye to infinities" and urged that Einstein's objections to quantum mechanics be seriously considered anew. Mr Pisello does just that in so far as he retakes "Einstein's insight" in the direction of unified field theories. He fails, however, to display a satisfactory theory, thus joining many others in this particular domain. The author also clearly shares with Einstein a socialist point of view upon the world; this does not justify the bloated language used by the publishers in the presentation of the book: "Pisello agrees with Einstein that quantum mechanics is a reactionary theory devised to maintain the mechanical view". Trying to get too close to Einstein, you may burn yourself up.

Failing to present clearly physical ideas and political analysis may be a consequence of the deep social frustrations experienced by Mr Pisello's generation of physicists. Trained in high energy particle physics, they got their degrees in America at the time of the Vietnam war. Their belief in 'pure' science was shaken when many of their peers were exposed as direct advisors for the US Department of Defence, and they could not get steady work as physicists in a tight competitive job market. This generation of physicists remains anxiously in search of the social and historical connections of scientific work.

Maurice Bazin

Maurice Banzin is Visiting Professor of Physics at the University of Campinas in Brazil.

\section{Sociology of science}

Science and the Sociology of Knowledge. Controversies of Sociology. Vol 8. By M. Mulkay. Pp. 132. (George Allen \& Unwin: London, 1979.) £7.50; paperback £3.50.

FROM the time of the 'founding fathers' of sociology in the nineteenth century, sociologists have been interested in the way in which bodies of knowledge are influenced by the social and cultural contexts in which they are produced. This is the sociology of knowledge. But scientific knowledge was always seen as something apart, special, and essentially independent of external social influences in a way that was not true of other specialised bodies of thought and knowledge. Thus, when a sociology of science emerged, and developed (quite rapidly from the 1950s), it became something other than the attempt to explain the content of scientific knowledge in terms of social structures and processes. The sociology of science developed as the attempt to understand the social conditions which made scientific progress possible: that is, it was concerned with general social and political conditions (giving rise to the idea of correspondence between science and democracy), as well as with the way in which scientific communities worked. There was a lot of interest in the 'normative structure' of science: the rules or moral code governing the ways in which scientists worked and interacted with each other.

A good deal of work in sociology of science is still being done along these lines, some of the most interesting of it concerned with the emergence and functioning of scientific communities in smaller or less industrialised nations. But Michael Mulkay is concerned with something else. His argument is that both this approach as well as discounting of the possibility of a sociology of scientific knowledge are founded upon a particular philosophical view of the nature of science, which he calls "the standard view of scientific knowledge”. Roughly speaking, this posits a real, objective, and uniform naturai world; it assumes that science, through unbiased and detached observation, can formulate laws of nature which describe this world; that science makes use of entirely objective criteria for evaluating knowledge claims, such that science is ultimately free of bias; and that theoretical laws are of a different status to empirical ones and stand in a quite different relationship to the natural world. He goes on to argue that this philosophy of science is no longer acceptable, and that it is therefore legitimate to try to establish a sociology of scientific knowledge.

Mulkay characterises the "new philosophy of science" as showing the dependence of all observation on theory, of all factual statements on (arbitrary) linguistic frameworks. All meaning in science becomes the product of negotiated agreement and is embeded in some changing framework of values and assumptions. Feyerabend's "epistemological anarchism" is an extreme version of all this.

There have now been a few sociological studies of science which made use of this relativised version of science, and Mulkay describes some of them. It becomes important to try to understand how scientists 'negotiate' 'what counts' as the 